ESAIM: PROCEEDINGS, December 2003, Vol. 13, 31-40

J.P. Penot, Editor

DOI: $10.1051 /$ proc:2003002

\title{
LA CONVEXITÉ GÉNÉRALISÉE EN ÉCONOMIE MATHÉMATIQUE
}

\author{
Crouzeix J.-P. ${ }^{1}$
}

\begin{abstract}
We show that generalized convexity appears quite naturally in some models of mathematical economics, specially in the consumer's behaviour theory.
\end{abstract}

Résumé. Le but de cet exposé est de montrer, à travers l'exemple de la théorie du consommateur, que la convexité généralisée est une notion essentielle et tout à fait naturelle en économie mathématique.

\section{INTRODUCTION}

La situation la plus simple où apparait la convexité généralisée en économie mathématique se rencontre dans la théorie du consommateur. Il s'agit pour celui-ci de déterminer son choix parmi un ensemble de biens disponibles de manière à satisfaire au mieux ses besoins, ses envies, tout en respectant une contrainte de budget. Le problème s'écrit

$$
\max _{x}[u(x): x \in X,\langle\hat{p}, x\rangle \leq b]
$$

où $X \subset \mathbb{R}^{n}$ représente l'ensemble des biens disponibles, le vecteur $x$ le choix fait par le consommateur, la composante $x_{i}$ désignant la quantité du bien $i, \hat{p}_{i}>0$ le prix d'une unité du bien $i,\langle\hat{p}, x\rangle=\hat{p}_{1} x_{1}+\hat{p}_{2} x_{2}+\cdots+\hat{p}_{n} x_{n}$ le coût du choix, $b>0$ le budget du consommateur, enfin la fonction $u$, appelée fonction d'utilité, mesure la satisfaction du consommateur.

Pour simplifier, nous supposerons dans tout cet exposé que $X$ est l'orthant positif $\mathbb{R}_{+}^{n}$. Il est naturel de demander à la fonction $u$ d'être strictement croissante, c'est-à- dire, telle que

$$
\begin{gathered}
x_{i} \leq y_{i} \forall i \Longrightarrow u(x) \leq u(y) \quad \text { et } \\
x_{i} \leq y_{i} \forall i \text { et } x \neq y \Longrightarrow u(x)<u(y) .
\end{gathered}
$$

Soit $k: u(X) \rightarrow \mathbb{R}$ une fonction continue strictement croissante et $\hat{u}(x)=k[u(x)]$. Le problème $(U)$ est alors totalement équivalent au problème

$$
\max _{x}[\hat{u}(x): x \in X,\langle\hat{p}, x\rangle \leq b]
$$

La représentation des préférences du consommateur par une fonction d'utilité n'est donc point unique.

Sans perte de généralité, on peut normaliser le vecteur prix en posant $p_{i}=b^{-1} \hat{p}_{i}$. Le problème devient

$$
v(p)=\max _{x}[u(x): x \in X,\langle p, x\rangle \leq 1] .
$$

La fonction $v$ est appelée la fonction d'utilité indirecte associée à $u$ qui, en conséquence, sera appelée fonction d'utilité directe. Il est clair que $v$ est décroissante sur $\mathbb{R}_{+}^{n}$.

1 LIMOS, Université Blaise Pascal, 63170 Aubière, e-mail: jp.crouzeix@math.univ-bpclermont.fr 
D'autre part,

$$
v(p) \leq \lambda \Longleftrightarrow[\langle p, x\rangle \leq 1 \Longrightarrow u(x) \leq \lambda] \Longleftrightarrow[u(x)>\lambda \Longrightarrow\langle p, x\rangle>1]
$$

et donc, en posant $[u>\lambda]=\{x: u(x)>\lambda\}$, pour tout $\lambda$ l'ensemble

$$
\{p: v(p) \leq \lambda\}=\bigcap_{x \in[u>\lambda]}\{p:\langle p, x\rangle>1\}
$$

est convexe comme intersection de demi-espaces ouverts. On voit donc apparaitre tout naturellement une structure de convexité sur la fonction d'utilité indirecte portant sur les ensembles de niveau de la fonction et non sur l'épigraphe comme pour les fonctions convexes. On parlera de convexité généralisée.

Il existe une dualité entre fonctions d'utilité directes et indirectes, en effet, sous certaines hypothèses tout à fait réalistes d'un point de vue économique, on a

$$
u(x)=\min _{p}[v(p): p \geq 0,\langle p, x\rangle \leq 1] .
$$

En raison de la symétrie entre les problèmes $(\mathrm{P})$ et $(\mathrm{Q})$, la fonction d'utilité directe $u$ sera, elle aussi, munie d'une structure de type convexité. Nous reviendrons sur ce point plus tard.

\section{Convexité GÉNÉRAliséE : DÉFinitions}

Soit $E$ un espace vectoriel topologique sur $\mathbb{R}$ et $f: E \rightarrow[-\infty,+\infty]$, on définit l'épigraphe et l'épigraphe strict de $f$ par

$$
\operatorname{epi}(f)=\{(x, \lambda): f(x) \leq \lambda\}, \quad \widetilde{\operatorname{epi}}(f)=\{(x, \lambda): f(x)<\lambda\}
$$

et pour tout $\lambda \in \mathbb{R}$ les ensembles de niveau $\lambda$ de $f$ par

$$
S_{\lambda}(f)=\{x: f(x) \leq \lambda\}, \quad \widetilde{S}_{\lambda}(f)=\{x: f(x)<\lambda\} .
$$

Le domaine de $f$ est l'ensemble $\operatorname{dom}(f)=\{x: f(x)<+\infty\}$.

Rappelons que, par définition, $f$ est dite convexe si epi $(f)$ (ou, de façon équivalente, si epi $(f)$ ) est un convexe de $E \times \mathbb{R}$. La fonction $f$ est convexe s.c.i. si et seulement si epi $(f)$ est un convexe fermé. Nous dirons que $f$ est quasiconvexe si tous les ensembles de niveau $S_{\lambda}(f), \lambda \in \mathbb{R}$ (ou, de façon équivalente, si tous les ensembles $\left.\widetilde{S}_{\lambda}(f), \lambda \in \mathbb{R}\right)$ sont des convexes de $E$. La fonction $f$ sera quasiconvexe s.c.i. si, et seulement si, tous les $S_{\lambda}(f)$ sont des convexes fermés. Le domaine d'une fonction quasiconvexe est convexe. Une fonction convexe est quasiconvexe.

Un convexe fermé est, en raison des théorèmes de séparation, une intersection de demi-espaces fermés. Ceci est une des clefs de l'analyse convexe, à partir de ce résultat, on définit la conjugaison de Fenchel, le sousdifférentiel, ... . Pour les fonctions quasiconvexes, on utilise une notion un peu plus fine : un ensemble $C \subset E$ est dit être uniment convexe ("evenly convexe" selon la terminologie de Fenchel) s'il est une intersection de demi-espaces ouverts. Les convexes ouverts, en raison des théorèmes de séparation, mais aussi les convexes fermés, puisque un demi-espace fermé est intersection de demi-espaces ouverts, sont des ensembles uniment convexes. Une fonction $f$ est dite être uniment quasiconvexe si ses ensembles de niveau sont uniment convexes. Ainsi les fonctions d'utilité indirectes sont uniment quasiconvexes. Les convexes ouverts et les convexes fermés étant uniment convexes, les fonctions quasiconvexes s.c.i. et les fonctions quasiconvexes s.c.s. sont uniment quasiconvexes. La notion d'uniment quasiconvexité est donc une notion rattachée à la continuité.

Il découle des définitions que $f$ est convexe si, et seulement si,

$$
\left[x_{1}, x_{2} \in E, t_{1}, t_{2}>0 \text { et } t_{1}+t_{2}=1\right] \Rightarrow f\left(t_{1} x_{1}+t_{2} x_{2}\right) \leq t_{1} f\left(x_{1}\right)+t_{2} f\left(x_{2}\right)
$$


et est quasiconvexe si et seulement si

$$
\left[x_{1}, x_{2} \in E, t_{1}, t_{2}>0 \text { et } t_{1}+t_{2}=1\right] \Rightarrow f\left(t_{1} x_{1}+t_{2} x_{2}\right) \leq \max \left[f\left(x_{1}\right), f\left(x_{2}\right)\right] .
$$

\subsection{Caractérisations}

Pour tout point $a \in E$ et toute direction $d \in E$ on définit

$$
f_{a, d}(t)=f(a+t d), \quad t \in \mathbb{R} .
$$

Il est facile de voir que $f$ est convexe (quasiconvexe) sur $E$ si, et seulement si, pour tout $(a, d) \in E^{2}$ la fonction d'une variable réelle $f_{a, d}$ est convexe (quasiconvexe) sur $\mathbb{R}$. Ainsi, comme pour les fonctions convexes, les caractérisations des fonctions quasiconvexes se dérivent des caractérisations des fonctions quasiconvexes d'une variable réelle.

Une fonction $f: \mathbb{R} \rightarrow[-\infty,+\infty]$ est quasiconvexe si, et seulement si, il existe $\bar{x} \in[-\infty,+\infty]$ tel que $f$ est décroissante sur $[-\infty, \bar{x}]$ et puis croissante sur $] \bar{x},+\infty]$ ou bien est décroissante sur $[-\infty, \bar{x}[$ puis croissante sur $[\bar{x},+\infty]$. En particulier une fonction monotone croissante ou décroissante sur un intervalle de $\mathbb{R}$ est quasiconvexe. Une fonction quasiconvexe n'est donc pas nécessairement continue sur l'intérieur de son domaine. C'est une différence majeure entre fonctions quasiconvexes et convexes.

Les caractérisations du premier ordre sont faciles à obtenir.

Proposition 2.1. Soit $C$ un convexe de $\mathbb{R}^{n}$ et soit $f: C \rightarrow \mathbb{R}$ différentiable. Alors chacune des conditions suivantes

$$
\begin{array}{ccc}
x, y \in C \text { et } f(y)<f(x) & \Longrightarrow & \langle\nabla f(x), y-x\rangle \leq 0, \\
x, y \in C \text { et } f(y) \leq f(x) & \Longrightarrow & \langle\nabla f(x), y-x\rangle \leq 0, \\
x, y \in C \text { et }\langle\nabla f(x), y-x\rangle>0 & \Longrightarrow \quad\langle\nabla f(y), y-x\rangle \geq 0
\end{array}
$$

est une condition nécessaire et suffisante pour que $f$ soit quasiconvexe sur $C$.

Si $f$ est différentiable, quasiconvexe et si $\nabla f(x)=0, f$ n'atteint pas nécessairement son minimum en $x$ : considérer, par exemple, la fonction d'une variable réelle $\theta$ définie par $\theta(t)=t^{3}$ au point $t=0$. On dira qu'une fonction différentiable $f$ est pseudoconvexe sur le convexe $C$ si

$$
x, y \in C \text { et } f(y)<f(x) \Longrightarrow\langle\nabla f(x), y-x\rangle<0 .
$$

Si $f$ est pseudoconvexe et $\nabla f(x)=0$ alors $f$ a un minimum global en $x$ et tout minimum local est aussi global. Une fonction pseudoconvexe est quasiconvexe. Réciproquement,

Proposition 2.2. Soit $C$ un convexe ouvert de $\mathbb{R}^{n}$ et $f: C \rightarrow \mathbb{R}$ différentiable. Alors $f$ est pseudoconvexe sur $C$ si et seulement si $f$ est quasiconvexe sur $C$ et a un minimum local en tout $x \in C$ tel que $\nabla f(x)=0$.

Les caractérisations du second ordre sont plus difficiles à obtenir (tout au moins en ce qui concerne les conditions suffisantes). On a le résultat suivant :

Théorème 2.1. Soit $C$ un convexe ouvert de $\mathbb{R}^{n}$ et $f: C \rightarrow \mathbb{R}$ deux fois continûment différentiable. Alors $f$ est pseudoconvexe sur $C$ si, et seulement si, les deux conditions suivantes sont satifaites :

a) $\left\langle\nabla^{2} f(x) h, h\right\rangle \geq 0$ pour tous $x \in C$ et $h$ tels que $\langle\nabla f(x), h\rangle=0$;

b) $f$ a un minimum local en tout $x$ tel que $\nabla f(x)=0$.

On remarque la différence avec la condition du second ordre pour la convexité : ici, on demande à la forme quadratique $\left\langle\nabla^{2} f(x) h, h\right\rangle$ d'être semi-définie positive seulement sur le sous-espace orthogonal au gradient et non sur l'espace tout entier. 
Si $\nabla f(x) \neq 0$, la condition a) est équivalente à dire que la matrice dite "bordée"

$$
\left(\begin{array}{cc}
\nabla^{2} f(x) & \nabla f(x) \\
(\nabla f(x))^{T} & 0
\end{array}\right)
$$

a exactement une valeur propre négative. Cette condition est souvent exprimée à partir des changements de signe dans la suite des déterminants des mineurs principaux de la matrice bordée. Notons que la matrice $\nabla^{2} f(x)$ peut avoir au plus une valeur propre négative.

Une revue récente des caractérisations du premier ordre et du second ordre, incluant les références historiques, est donnée dans [9], voir aussi [10].

\section{Convexifiabilité}

Si $f$ est convexe et si $k$ est une fonction numérique continue strictement croissante alors $k \circ f$ est quasiconvexe et, lorsque différentiable, pseudoconvexe. On peut se poser le problème inverse : étant donnée une fonction $f$ quasiconvexe, est ce qu'il existe une fonction numérique continue strictement croissante $k$ telle que $k \circ f$ soit convexe? Une telle fonction sera alors appelée convexifiable ou encore convexe transformable. La réponse est non : une fonction aussi simple que la fonction $f\left(x_{1}, x_{2}\right)=x_{2}^{-1} x_{1}$ n'est point convexifiable sur $C$ quel que soit $C$ convexe ouvert contenu dans $] 0, \infty\left[{ }^{2}\right.$. Le problème de la convexifiabilité est un problème assez difficile. On pourra se reporter aux références [20], [21] et [3].

Il est un cas où on peut donner une réponse facile, c'est le cas où $f$ est deux fois continûment différentiable dans un voisinage d'un point $\bar{x}$ et où la matrice $\nabla^{2} f(\bar{x})$ est définie positive sur le sous-espace orthogonal à $\nabla f(\bar{x})$. Il existe alors $r>0$ tel que la fonction $g(x)=\exp (r f(x))$ soit convexe sur un voisinage convexe de $\bar{x}$. On dira que $f$ est $r$-convexe sur ce voisinage, voir par exemple [10].

\section{Retour SUR Les Fonctions D'Utilité}

La fonction d'utilité est une représentation numérique des préférences du consommateur. Celles-ci sont exprimées au moyen d'un préordre : étant donnés $x, y \in \mathbb{R}_{+}^{n}, x \preceq y$ signifie que $y$ est préféré à $x$ et $x \prec y$ signifie que $y$ est strictement préféré à $x$. Si le préordre est transitif, total (étant donnés $x, y$, alors ou bien $x \preceq y$, ou bien $y \preceq x$, ou les deux à la fois), et possède une propriété de continuité (pour tout $x$, l'ensemble $\{y: x \preceq y\}$ ( $\{y: y \preceq x\})$ est fermé), alors (voir par exemple [12]), il existe une fonction d'utilité s.c.s. (s.c.i.) $u$ telle que

$$
x \preceq y \Longleftrightarrow u(x) \leq u(y) .
$$

Nous supposons ici que le préordre est strictement croissant, c'est-à-dire, $x \preceq y$ lorsque $x, y \in \mathbb{R}_{+}^{n}, x \leq y$ et $x \prec y$ lorsque $x, y \in \mathbb{R}_{+}^{n}, x \leq y$ et $x \neq y$. Etant donné un vecteur prix $\hat{p}$ appartenant à l'orthant strictement positif, en raison de la continuité de la fonction d'utilité, il existe pour le consommateur une consommation optimale $x$. Plus exactement, pour tout $\hat{p}>0$ l'ensemble des solutions optimales $X(\hat{p})$ du problème ci-dessous est non vide

$$
v(\hat{p})=\max _{x}[u(x): x \in X,\langle\hat{p}, x\rangle \leq 1] .
$$

Puisque le préordre est strictement croissant, on a l'équivalence

$$
\hat{x} \in X(\hat{p}) \Longleftrightarrow[v(\hat{p})=u(\hat{x}) \text { et }\langle\hat{p}, \hat{x}\rangle=1] .
$$

Pour tout $p \geq 0$ tel que $\langle p, \hat{x}\rangle \leq 1$ on a

$$
v(p)=\max _{x}[u(x): x \in X,\langle p, x\rangle \leq 1] \geq u(\hat{x}),
$$


donc

et finalement,

$$
v(\hat{p})=u(\hat{x}) \leq \inf _{p}[v(p): p \geq 0,\langle p, \hat{x}\rangle \leq 1] \leq v(\hat{p})
$$

$$
u(\hat{x})=\min _{p}[v(p): p \geq 0,\langle p, \hat{x}\rangle \leq 1] .
$$

On voit ainsi apparaître la dualité annoncée entre fonctions d'utilité directes et indirectes. Mais nous n'avons obtenu cette relation que pour les $x$ solutions optimales du problème $(P)$ pour un vecteur prix $p$. Disons ici que sous certaines hypothèses de continuité, de croissance, . ., sur la fonction $u$ la relation $(Q)$ a lieu. En raison de la parfaite symétrie entre $u$ et $v$, le min étant remplacé par max, $u$ doit être uniment quasiconcave. Les lecteurs intéressés se reporteront aux références suivantes [27], [23], [14], [4], [24], [26] et [25] et les références incluses. Signalons qu'une dualité du même type existe en théorie de la production, voir par exemple [14].

\section{LA CORRESPONDANCE DEMANDE}

Nous supposons que $u$ est quasiconcave strictement croissante sur $] 0,+\infty\left[{ }^{n}, v\right.$ quasiconvexe strictement décroissante sur $] 0,+\infty\left[{ }^{n}\right.$ et que l'on a pour tous $\left.x, p \in\right] 0, \infty\left[{ }^{n}\right.$

$$
\begin{aligned}
& v(p)=\max _{y}[u(y): y \geq 0,\langle p, y\rangle=1], \\
& u(x)=\min _{q}[v(q): q \geq 0,\langle q, x\rangle=1] .
\end{aligned}
$$

Désignons par $X(p)$ et $P(x)$ les ensembles de solutions optimales de ces problèmes. Ces ensembles sont convexes et, sous réserve de la continuité des fonctions $u$ et $v$ sur $\mathbb{R}_{+}^{n}$, fermés. Il est clair que l'on a les équivalences suivantes :

$$
x \in X(p) \Longleftrightarrow[v(p)=u(x) \text { et }\langle p, x\rangle=1] \Longleftrightarrow p \in P(x)
$$

La multiapplication (correspondance) $X$ est appelée demande. Il est clair que les correspondances $X$ et $P$ sont inverses l'une de l'autre. De la même façon que la relation entre fonctions d'utilité directe et indirecte doit être comparée à la relation entre une fonction convexe $f$ et sa conjuguée $f^{*}$, la relation entre $X$ et $P$ doit être comparée à la relation entre les sous-différentiels $\partial f$ et $\partial f^{*}$ d'une fonction convexe et de sa conjuguée.

Supposons que l'on a $\hat{p}>0, X(\hat{p})$ réduit au singleton $\{\hat{x}\}$ avec $\hat{x}>0, u$ continûment différentiable dans un voisinage de $\hat{x}$ et $\langle\hat{x}, \nabla u(\hat{x})\rangle \neq 0$ (on a alors $\hat{x}^{t} \nabla u(\hat{x})>0$ ). On montre alors que $P(\hat{x})$ est réduit au singleton $\{\hat{p}\}$ et $v$ est continûment différentiable dans un voisinage de $\hat{p}$. Il existe donc deux fonctions univoques $x(p)$ et $p(x)$ définies sur des voisinages de $\hat{p}$ et $\hat{x}$ respectivement telles que sur ces voisinages on ait $X(p)=\{x(p)\}$ et $P(x)=\{p(x)\}$. On peut alors montrer, à partir des conditions d'optimalité dans les problèmes $(\mathrm{P})$ et $(\mathrm{Q})$, que l'on a

$$
\begin{gathered}
x(p)=\frac{\nabla v(p)}{\mu(p)} \quad \text { et } \quad p(x)=\frac{\nabla u(x)}{\lambda(x)} \text { avec } \\
0<\lambda(x)=\langle x, \nabla u(x)\rangle=-\langle p, \nabla v(p)\rangle=-\mu(p) .
\end{gathered}
$$

Supposons en outre $u$ et $v$ deux fois différentiables, alors

$$
\left(\begin{array}{cc}
\frac{\nabla^{2} v(p)}{\mu(p)} & -x \\
-x^{t} & 0
\end{array}\right)=\left(\begin{array}{cc}
I & x \\
p^{t} & 0
\end{array}\right)\left(\begin{array}{cc}
\frac{\nabla^{2} u(x)}{\lambda(x)} & -p \\
-p^{t} & 0
\end{array}\right)^{-1}\left(\begin{array}{cc}
I & p \\
x^{t} & 0
\end{array}\right)
$$

sous réserve, bien sûr, que la matrice inverse de la formule ci-dessus existe. Cette relation fait pendant à la relation sur les hessiens d'une fonction convexe et de sa conjuguée, lorsque $x^{*}=\nabla f(x)$ on a

$$
\nabla^{2} f^{*}\left(x^{*}\right) \nabla^{2} f(x)=I
$$


Sous les mêmes hypothèses, la fonction demande $x$ est différentiable dans un voisinage de $p$ et sa matrice jacobienne $x^{\prime}(p)$ peut être calculée en résolvant le système linéaire

$$
\left(\begin{array}{cc}
\nabla^{2} u(x) & -p \\
-p^{t} & 0
\end{array}\right)\left(\begin{array}{c}
x^{\prime}(p) \\
\nabla^{t} \lambda(x)
\end{array}\right)=\left(\begin{array}{c}
\lambda(x) I \\
x^{t}
\end{array}\right)
$$

On remarquera que l'expression de $x(p)$ dans l'équation (3) et celle de $x^{\prime}(p)$ dans l'équation (6) ne dépendent pas de la fonction d'utilité choisie pour représenter le préordre : si on remplace $u$ par $k \circ u$ avec $k$ (deux fois) différentiable et $k^{\prime}$ strictement positif, on obtient le même résultat pour $x(p)$ et $x^{\prime}(p)$.

\section{SÉPARABiLitÉ}

Les fonctions quasiconvexes, comme les fonctions convexes, possèdent certaines propriétés importantes, en particulier

- si les fonctions $f_{i}: X \rightarrow[-\infty, \infty], i \in I, I$ famille d'indices arbitraire, sont quasiconvexes alors $\sup _{i} f_{i}$ est quasiconvexe ;

- si $\varphi$ est quasiconvexe sur l'espace vectoriel produit $X \times Y$, alors la fonction performance définie par $h(x)=\inf [\varphi(x, y): y \in Y]$ est quasiconvexe sur $X$;

- si $f$ est quasiconvexe sur $X$ et $k$ est une fonction numérique croissante, alors $k \circ f$ est quasiconvexe sur $X$.

En revanche, la somme de deux fonctions quasiconvexes sur un même espace $X$ n'est pas quasiconvexe en général. Considérer, par exemple, la fonction $f(x)=-[\exp (x)+\exp (-x)]$ qui est la somme de deux fonctions monotones donc quasiconvexes.

De nombreux économistes se sont penchés sur le problème de l'additivité séparable, c'est-à-dire sur le problème de la quasiconcavité d'une fonction $u$ définie sur un ensemble produit $C=C_{1} \times C_{2} \times \cdots \times C_{p}$ par une somme de fonctions d'utilité

$$
u\left(x_{1}, x_{2}, \cdots, x_{p}\right)=u_{1}\left(x_{1}\right)+u_{2}\left(x_{2}\right)+\cdots+u_{p}\left(x_{p}\right)
$$

où $u_{i}: C_{i} \rightarrow \mathbb{R}, C_{i} \subset \mathbb{R}^{n_{i}}$. Les indices $i$ correspondent, par exemple, à des périodes différentes, à des biens de natures différentes. On supposera ici que les $C_{i}$ sont des ensembles convexes ouverts non vides et que le problème est non dégénéré : $p \geq 2$ et pour tout $i$ la fonction $u_{i}$ est non constante $\operatorname{sur} C_{i}$.

L'hypothèse de quasiconcavité sur $u$ est très contraignante, on peut en effet alors montrer que toutes les fonctions $u_{i}$ sont continues, concaves sauf peut être une, et encore celle ci doit être $r$-concave .

A noter que, en passant aux exponentielles, la séparabilité additive correspond à la séparabilité multiplicative

$$
\hat{u}\left(x_{1}, x_{2}, \cdots, x_{p}\right)=\hat{u}_{1}\left(x_{1}\right) \hat{u}_{2}\left(x_{2}\right) \cdots \hat{u}_{p}\left(x_{p}\right) .
$$

On montre alors que $\hat{u}$ est quasiconcave si toutes les fonctions $u_{i}$ sont log-concaves sauf peut être une, et celle ci doit être log-concave transformable.

Un exemple classique de fonctions d'utilité séparables est celui des fonctions de Cobb-Douglas. Sous leur forme la plus simple, elles sont donnés par

$$
u\left(x_{1}, x_{2}, \cdots, x_{p}\right)=x_{1}^{\alpha_{1}} x_{2}^{\alpha_{2}} \cdots x_{p}^{\alpha_{p}} .
$$

Sous une forme plus élaborée, elles sont de la forme

$$
u\left(x_{1}, x_{2}, \cdots, x_{p}\right)=\left[u_{1}\left(x_{1}\right)\right]^{\alpha_{1}}\left[u_{2}\left(x_{2}\right)\right]^{\alpha_{2}} \cdots\left[u_{p}\left(x_{p}\right)\right]^{\alpha_{p}} .
$$

Pour les résultats les plus complets sur le problème de la séparabilité, on consultera les références [13], [5], [32] et [6]. Voir aussi [7] et [8]. 


\section{Monotonie GÉnÉRALisÉe}

Retournons au schéma de dualité. Il est clair que si $x \in X(p)$ on a

$$
\begin{aligned}
& \langle p, y\rangle \leq 1=\langle p, x\rangle \quad \Longrightarrow \quad u(y) \leq u(x), \\
& \langle p, y\rangle<1=\langle p, x\rangle \quad \Longrightarrow \quad u(y)<u(x) .
\end{aligned}
$$

Par suite, on ne peut avoir en même temps

$$
x_{1} \in X\left(p_{1}\right), x_{2} \in X\left(p_{2}\right),\left\langle p_{1}, x_{2}-x_{1}\right\rangle \leq 0 \text { et }\left\langle p_{2}, x_{1}-x_{2}\right\rangle<0 .
$$

Donc

et

$$
\left.\begin{array}{c}
x_{1} \in X\left(p_{1}\right), x_{2} \in X\left(p_{2}\right), \\
\left\langle p_{1}, x_{2}-x_{1}\right\rangle \leq 0
\end{array}\right\} \Longrightarrow\left\langle p_{2}, x_{2}-x_{1}\right\rangle \leq 0
$$

$$
\left.\begin{array}{c}
x_{1} \in X\left(p_{1}\right), x_{2} \in X\left(p_{2}\right), \\
\left\langle p_{1}, x_{2}-x_{1}\right\rangle<0
\end{array}\right\} \Longrightarrow\left\langle p_{2}, x_{2}-x_{1}\right\rangle<0 .
$$

En fait, on a plus

$$
\left.\begin{array}{cl}
x_{i} \in X\left(p_{i}\right) & \forall i=1,2, \cdots, q \\
\left\langle p_{i}, x_{i+1}-x_{i}\right\rangle \leq 0 & \forall i=1,2, \cdots, q-1
\end{array}\right\} \Longrightarrow\left\langle p_{q}, x_{1}-x_{q}\right\rangle \geq 0
$$

et

$$
\left.\begin{array}{cc}
x_{i} \in X\left(p_{i}\right) \quad \forall i=1,2, \cdots, q \\
\left\langle p_{i}, x_{i+1}-x_{i}\right\rangle \leq 0 \quad \forall i=1,2, \cdots, q-1 \\
\text { une des inégalités étant stricte }
\end{array}\right\} \Longrightarrow\left\langle p_{q}, x_{1}-x_{q}\right\rangle>0 .
$$

On dit qu'une multiapplication $\Gamma: X \rightrightarrows X^{*}$ est quasimonotone si

$$
\left.\begin{array}{c}
x_{1}^{*} \in \Gamma\left(x_{1}\right), x_{2}^{*} \in \Gamma\left(x_{2}\right), \\
\left\langle x_{1}^{*}, x_{2}-x_{1}\right\rangle>0
\end{array}\right\} \Longrightarrow\left\langle x_{2}^{*}, x_{2}-x_{1}\right\rangle \geq 0 .
$$

et est pseudomonotone si

$$
\left.\begin{array}{c}
x_{1}^{*} \in \Gamma\left(x_{1}\right), x_{2}^{*} \in \Gamma\left(x_{2}\right), \\
\left\langle x_{1}^{*}, x_{2}-x_{1}\right\rangle>0
\end{array}\right\} \Longrightarrow\left\langle x_{2}^{*}, x_{2}-x_{1}\right\rangle>0 .
$$

On voit donc que, en terme de fonctions d'utilité et de correspondances de demande, $-P=-X^{-1}$ est pseudomonotone. De plus, la relation (9) signifie que cette pseudomonotonicité a un caractère cyclique. En travaillant avec la fonction d'utilité indirecte $v$ au lieu de $u$ on aurait tout aussi bien montré que $-X=-P^{-1}$ était pseudomonotone et cycliquement pseudomonotone, ce que l'on peut aussi dériver de la pseudomonotononie de $-P=-X^{-1}$ et du fait que si $x \in X(p)$ on a $\langle p, x\rangle=1$.

Supposons maintenant que $f$ soit une fonction quasiconvexe. En tout $x \in \operatorname{dom}(f)$, on définit le cône normal $N(x)$ par

$$
x^{*} \in N(x) \Longleftrightarrow\left[f(y) \leq f(x) \Rightarrow\left\langle x^{*}, y-x\right\rangle \leq 0\right] .
$$

Il s'ensuit que l'on ne peut avoir en même temps

$$
x_{1}^{*} \in N\left(x_{1}\right), x_{2}^{*} \in N\left(x_{2}\right),\left\langle x_{1}^{*} x_{2}-x_{1}\right\rangle>0 \text { et }\left\langle x_{2}^{*} x_{1}-x_{2}\right\rangle>0 .
$$

On en déduit que $N$ est quasimonotone sur $\operatorname{dom}(f)$. Supposons en outre que $N$ vérifie la condition

$$
\left[x^{*} \in N(x), x^{*} \neq 0 \text { et } f(y)<f(x)\right] \Longrightarrow\left\langle x^{*}, y-x\right\rangle<0 .
$$

Cette condition est vérifiée lorsque $f$ est s.c.s. . Sous cette condition,

$$
\left\langle x^{*}, y-x\right\rangle \geq 0 \Longrightarrow f(y) \geq f(x) .
$$


On obtient alors

$$
\left.\begin{array}{cc}
x_{i}^{*} \in N\left(x_{i}\right) & \forall i=1,2, \cdots, q \\
\left\langle x_{i}^{*}, x_{i+1}-x_{i}\right\rangle \geq 0 \quad \forall i=1,2, \cdots, q-1 \\
\text { une des inégalités étant stricte }
\end{array}\right\} \Longrightarrow\left\langle x_{q}^{*}, x_{1}-x_{q}\right\rangle<0
$$

$N$ est alors cycliquement pseudomonotone.

Bien des sous-différentiels ont été définis pour les fonctions quasiconvexes. Une des propriétés minimales qu'ils doivent vérifier est que les cônes qu'ils engendrent correspondent aux cônes normaux. Ces sous-différentiels seront ainsi quasimonotones (cycliquement pseudomonotones). Le cas le plus simple est celui où la fonction $f$ est différentiable : si $f$ est quasiconvexe alors $\nabla f$ doit être quasimonotone, si $f$ est pseudoconvexe alors $\nabla f$ doit être cycliquement pseudomonotone.

\section{INTÉGRABILITÉ : LES PRÉFÉRENCES RÉVÉLÉES}

Les données obtenues à partir de l'observation du comportement d'un consommateur sont ses choix (ses préférences) lorsqu'il dispose du budget $b$ et que le vecteur des prix unitaires est $p$. Après normalisation des prix, l'observation conduit ainsi à la correspondance demande $X$ et non à une fonction d'utilité que nous savons d'ailleurs ne pas être définie de façon unique. Le problème se pose donc de reconstituer à partir de la correspondance demande $X$ une fonction d'utilité. Ce problème est connu comme le problème des préférences révélées.

Supposons tout d'abord que, pour $p \in] 0, \infty\left[{ }^{n}, X(p)\right.$ est réduit au singleton $\left.\{x(p)\} \subset\right] 0, \infty\left[{ }^{n}\right.$. Supposons en outre que $x$ est continûment différentiable sur $] 0,+\infty\left[{ }^{n}\right.$. A partir des équations

$$
\langle x(p), p\rangle=1 \text { et } \nabla v(p)=\langle\nabla v(p), p\rangle x(p)
$$

on déduit

$$
\begin{gathered}
\left(I-x(p) p^{t}\right) \frac{\nabla^{2} v(p)}{\langle\nabla v(p), p\rangle}=x^{\prime}(p)+x(p) x^{t}(p), \\
\left(I-x(p) p^{t}\right) \frac{\nabla^{2} v(p)}{\langle\nabla v(p), p\rangle}\left(I-p x^{t}(p)\right)=x^{\prime}(p)\left(I-p x^{t}(p)\right)
\end{gathered}
$$

et, puisque $\left(I-x(p) p^{t}\right)^{2}=\left(I-x(p) p^{t}\right)$,

$$
\left(I-x(p) p^{t}\right) \frac{\nabla^{2} v(p)}{\langle\nabla v(p), p\rangle}\left(I-p x^{t}(p)\right)=\left(I-x(p) p^{t}\right) x^{\prime}(p)\left(I-p x^{t}(p)\right) .
$$

Rappelons la relation (5) liant $\nabla^{2} v(p)$ à $\nabla^{2} u(x)$. On voit qu'une condition nécessaire pour l'existence d'une fonction d'utilité deux fois différentiable est la symétrie de la matrice $\left(I-x(p) p^{t}\right) x^{\prime}(p)\left(I-p x^{t}(p)\right)$. De plus, puisque $v$ doit être quasiconvexe, on a

$$
\langle\nabla v(p), h\rangle=0 \Longrightarrow\left\langle\nabla^{2} v(p) h, h\right\rangle \geq 0 .
$$

Rappelons que l'on a $\langle\nabla v(p), p\rangle<0$ et que $x(p)$ est proportionnel à $\nabla v(p)$. On en déduit que la matrice $\left(I-x(p) p^{t}\right) x^{\prime}(p)\left(I-p x^{t}(p)\right)$ doit être semi-définie négative. Ces deux conditions peuvent s'exprimer en disant que la matrice $x^{\prime}(p)$ est symétrique et semi-définie négative sur le sous espace orthogonal à $x(p)$. Ces conditions correspondent aux conditions bien connues de symétrie et semi-définie négativité sur la matrice de Slutsky.

Le problème qui se pose est le suivant, étant donnée la correspondance $x$ satisfaisant ces deux conditions, est ce qu'il existe une fonction d'utilité deux fois différentiable correspondante ? Il n'y a aucune difficulté en dimension 2 (voir par exemple [30]). En dimension supérieure, il existe des démonstrations basées sur le calcul différentiel extérieur lorsque la fonction $x$ est analytique (voir par exemple [2] et [16]) et une approche toute 
récente ( [11]) dans le cas où $x$ est continûment différentiable. Ce problème doit être comparé au problème suivant, étant donnée une fonction $F: \mathbb{R}^{n} \rightarrow \mathbb{R}^{n}$ continûment différentiable telle que la matrice $F^{\prime}(x)$ est symétrique et semi-définie positive pour tout $x$, est ce qu'il existe une fonction $f$ convexe différentiable telle que $\nabla f=F$ ? On sait que la réponse est oui, $f$ est alors définie à une constante additive près. Dans notre cas $u$ sera définie à une fonction scalaire $k$ près, le problème est bien plus difficile.

Passons au cas général où la correspondance demande $X$ est multivoque. Nous avons vu que $-X$ doit être pseudomonotone (7) et même cycliquement pseudomonotone (9). Ces conditions sont connues par les économistes sous les noms de l'axiome faible et l'axiome fort des préférences révélées. Le problème est le suivant, étant donnée la demande $X$ est ce qu'il existe une fonction d'utilité $u$ correspondante ? Ce problème doit être comparé au problème suivant : étant donnée une multiapplication $F$, existe-t'il une fonction $f$ convexe telle que son sous-différentiel $\partial f$ coincide avec $F$ ? On sait que la réponse est oui lorsque $F$ est maximale cycliquement monotone ; $f$ est alors définie à une constante additive près, la monotonicité simple n'est point suffisante. Dans notre cas, la pseudomonotonie (i.e., l'axiome faible des préférences révélées) ne sera point suffisante, la cyclique pseudomonotonie sera exigée, la maximalité sera exprimée en disant que le graphe de $X$ doit être fermé et que pour tout $p, X(p)$ est un convexe fermé. Un travail récent sur ce sujet est la référence [15].

Il est clair que pour les fonctions demandes univoques différentiables, la symétrie et semi-définie négativité de la matrice de Slutsky correspondent à la cyclique pseudomonotonie, tout comme dans le cas convexe pour une application $F$ différentiable, la symétrie et semi-définie positivité des matrices $F^{\prime}(x)$ correspondent à la cyclique monotonie de $F$.

Voici quelques références parmi un grand nombre de travaux consacrés à ce sujet : [1], [18], [17], [28], [29], [19], [30] et la référence historique [31].

\section{COMMENT RESTER INFORMÉ ?}

Pour suivre les développements en convexité généralisée et obtenir plus de références, on pourra suivre les activités du 'Working group on generalized convexity". Pour cela, se connecter au site Internet : http://www.ec.unipi.it/ genconv/

On se reportera également aux différents proceedings édités à la suite des symposiums du groupe.

\section{REFERENCES}

[1] Afriat S. N., Demand functions and the Slutsky matrix, Princeton University Press, 1982.

[2] Chiappori P. A. and Ekeland I., Aggregation and Market Demand : an Exterior Differential Calculus Viewpoint, Econometrica 67, 1999, 1435-1458.

[3] Crouzeix J.-P., Contributions à l'étude des fonctions quasiconvexes, Thèse de doctorat es sciences, Université de Clermont 2, 1977.

[4] Crouzeix J.-P., Duality between direct and indirect utility functions, differentiabilities properties, Journal of Mathematical Economics 12, 1983, 149-165.

[5] Crouzeix J.-P. and Lindberg P. O., Additively decomposed quasiconvex functions, Mathematical Programming 35, $1986,42-57$.

[6] Crouzeix J.-P. et Kebbour R., Index multiplicatifs de convexité/concavité et applications, Cahiers du Centre de Recherche Opérationnelle (Bruxelles), Vol. 34, no 1, 1992, 7-24.

[7] Crouzeix J.-P. and Hassouni A., Quasimonotonicity of separable operators and monotonicity indices, SIAM Journal on Optimization 3, 1994, 649-658.

[8] Crouzeix J.-P. and Hassouni A., Generalized monotonicity of a separable product of operators: the multivalued case, Set-Valued Analysis 3, 1995, 351-373.

[9] Crouzeix J.-P., Characterizations of generalized convexity and generalized monotonicity, a survey, in Generalized convexity and generalized monotonicity, Crouzeix J.-P., Martinez-Legaz J.-E. and Volle M. eds, Non convex optimization and its applications, Kluwer Academic Publishers, 1998, 237-256.

[10] Crouzeix J.-P., Criteria for generalized convexity and generalized monotonicity in the differentiable case, to appear in the Handbook on generalized convexity, Kluwer Academic Publishers. 
[11] Crouzeix J.-P. and Rapcsak T., Pseudoconvex integration, technical paper 2003.

[12] Debreu G., Theory of value: an axiomatic approach of economic equilibrium, Wiley, New York, 1959.

[13] Debreu G. and Koopmans T. C., Additively decomposed quasiconvex functions, Mathematical Programming 24, 1982 , 1-38.

[14] Diewert W. E., Duality approaches to microeconomics theory, in Handbook of Mathematical Economics vol. 2, Arrow K. J. and Intrilligator M. D. eds, North Holland Publishing Company, 1982, 535-599.

[15] Eberhard A. and Crouzeix J.-P., Integration of a normal cone relation generated by the level sets of pseudoconvex functions, in process.

[16] Ekeland I., Exterior Differential Calculus and Applications to Economy Theory, CEREMADE, Université Paris Dauphine.

[17] Green J., Mas-Colell A. and Whinston M., Microeconomics theory, Oxford University Press, 1995.

[18] Houthakker H. S., Revealed preference and the utility function, Economica 17, 1950, 159-174.

[19] Hurwicz L. and Uzawa, On the integrability of demand functions, Ch. 6 in Preferences, Utility and Demand, Chipman J. Hurwicz L. and Sonnenscheim, Harcourt Brace Jovanovich, 1971.

[20] Kannai Y., Concavifiability and constructions of concave utility functions, Journ. of Mathematical Economics 4, $1974,1-56$.

[21] Kannai Y., Concave utility functions, existence, constructions and cardinality, in Generalized concavity in optimization and economics, Schaible S. and Ziemba W. T. eds, Academic Press, 1981, 543-612.

[22] Kihlstrom R., Mas Colell A. and Sonnenscheim H., The demand theory of the weak axiom of revealed preference, Econometrica 44, 1976, 971-978.

[23] Lau L. J., Duality and the structure of utility functions, Journal of Economic Theory 1, 1970, 374-396.

[24] Martínez-Legaz J.-E., Duality between direct and indirect utility functions under minimal hypothesis, Journal of Mathematical Economics 20, 1991, 199-209.

[25] Martínez-Legaz J.-E., Generalized convex duality and its economic applications, to appear in the Handbook on generalized convexity, Kluwer Academic Publishers.

[26] Penot J.-P., Observations about microeconomics and duality, International Workshop on Games, Mathematical Programming in Economics, Aix en Provence, décembre 2001.

[27] Roy B., De l'utilité, Paris, Hermann, 1942.

[28] Sakai Y., Equivalence of the weak and strong axioms of revealed preference without demand continuity assumptions: a "regularity condition" approach, Journal of Economic Theory 8, 1974, 292-304.

[29] Sakai Y., Revealed favorability, indirect utility and direct utility, Journal of Economic Theory 14, 1977, 113-129.

[30] Samuelson P. A., Consumption theory in terms of revealed preference, Economica, 1948, 243-253, also in Utility theory: a book of readings, A. N. Page editor, John Wiley \& Sons, 1968, 149-159.

[31] Slutsky E., Sulla theoria del bilancio del consumatore, Giornali degli economisti 51, 1915, 1-26.

[32] Yaari M. E., A note on separability and quasiconcavity, Econometrica 45, 1977, 1183-1186. 